\title{
3D object-based feature extraction from 3-stereo DSM in urban context
}

\author{
K. Kulessa \& S. Lang \\ Department of Geoinformatics - Z_GIS, University of Salzburg, Austria - (Kerstin.Kulessa, Stefan.Lang)@sbg.ac.at
}

KEY WORDS: 3D features, triple-stereo, Digital Surface Model, Pléiades, Semi-Global Matching, urban area

\begin{abstract}
:
For urban planning purposes updated information on land-use and urban structures and change dynamics are essential. Satellite Earth observation is a very effective information source, to provide actual representations of the situation 'as is' in regular time steps. The Pléiades constellation offers very high resolution imagery with a triple-stereo acquisition. The intrinsic combination of thematic and height information is an additional asset provided by recent VHR stereo imagery. Digital surface models (DSM) can be derived through stereo matching algorithms, one of which is semi-global matching (SGM). SGM integrates local and global matching methods with a short processing time and accurate surface generation.

We use the generated DSM for extracting 3-D features which enrich object-based class models of composite urban objects. These multi-purpose 3-D object feature datasets can be used for various planning and monitoring purposes, revealing height-related characteristics of urban objects (blocks, neighbourhoods, parks, industrial sites, etc.). In addition object-wise aggregated height information can be used to better assess the adherence of actual building activities to zoning or land development plans, e.g. with respect to maximum or average height in recreational areas etc.

As compared to detailed and potentially more accurate DSMs generated from LiDAR data, a (3-)stereo DSM has the advantage of recording height and spectral information instantaneously, so not to lose any information in high dynamic urban settings due to a time gap. Height and colour information, when integrated by spatial context and multi-scale OBIA methods, enable a wealth of new applications in urban feature characterisation and 3D city model applications.
\end{abstract}

\section{INTRODUCTION}

Urban areas are characterised by continuous change. This is a challenge for urban planning purposes because regularly updated information on the region is required to get an idea on the dynamics of urban structures and the changes in urban landuse. For these purposes it is important to have data with a high spatial resolution because the structure and the dynamics of the city have to be recorded in detail for urban planning, including information on the third dimension. Zhou (2013) used LiDAR heights to improve land-use classification. For urban areas, Laser scanning data, including the height information, is very accurate and has a very high spatial resolution which is also important for cities. A disadvantage of laser scanning data is the high cost and effort for acquisition of the data resulting in very accurate data sets that could not be updated too often due to high costs.

As opposed to this, satellite imagery is nowadays available in high temporal and spatial resolution. This data is already used for a variety of applications in urban areas such as for land-use classification or change detection analysis. Some satellites provide data in stereo or for some time past even tri-stereo acquisition mode. This mode can be used to derive height information from the satellite data with a high resolution which can be used as alternative to laser scanning data (Gehrke et al. 2010). Height information from tri-stereo satellite imagery can be calculated by stereo matching. The height information of this satellite DSM can be also used to improve classifications or even classify objects. This study shows the workflow of how to create a DSM from tri-stereo Pléiades images and explains the
3-D object extraction with eCognition software for statistical analysis in the field of urban planning.

\section{METHODS}

\subsection{Study Area}

This study has been done for the study area of Salzburg in Austria, covering the EuRegio greater region. The extent of the study area has a size of around $165 \mathrm{~km}^{2}$ and is characterised by a variety of topographies. Located north of the Alps, the city of Salzburg is situated in a rather flat basin bounded by the Gaisberg and characterized by some steep hills such as the Kapuzinerberg or Mönchsberg in the flat city area. Furthermore, the region of the study area is shaped by the Salzach River and the Saalach River, which forms the border between Austria and Germany and issues into the Salzach River in the north of the city (see Figure 1). The study area contains two cities: the city of Freilassing on the German side of the Saalach River, which mainly includes single family houses in a city environment of lower building density, and the larger city of Salzburg on the Austrian side. The city of Salzburg has a high building density with multi-story buildings in the inner city and a lower density in the residential areas. 


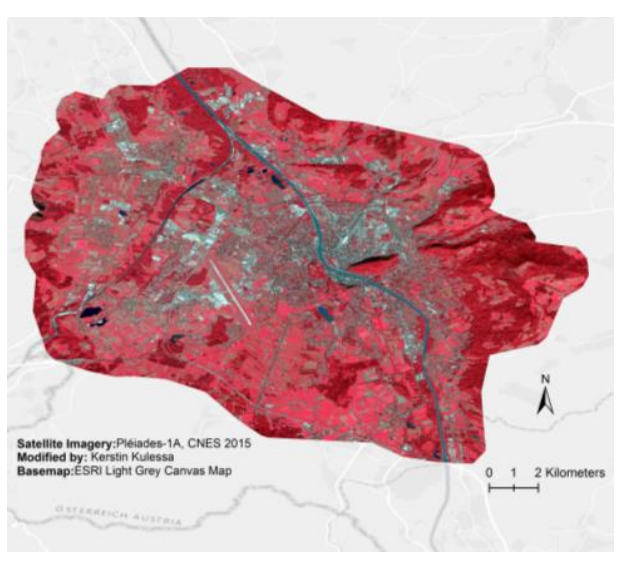

Figure 1. Pléiades false colour image showing the extent of the study area

\subsection{Data}

For this study there has been used Pléiades tri-stereo satellite imagery recorded during one pass. The Pléiades sysrem consists of the twin satellites Pléiades-1A launched in 2011 and Pléiades-1B launched in 2012. The two satellites, equipped with a pushbroom scanner, operate on the same orbit and are located $180^{\circ}$ from each other. This constellation enables a higher frequency of image acquisition which results in a larger temporal resolution. With a viewing angle of $+/-30^{\circ}$, which can be maximised up to $+/-47^{\circ}$ (ASTRIUM, 2012), a daily revisit of nearly every location on the Earth is possible (see Figure 2).

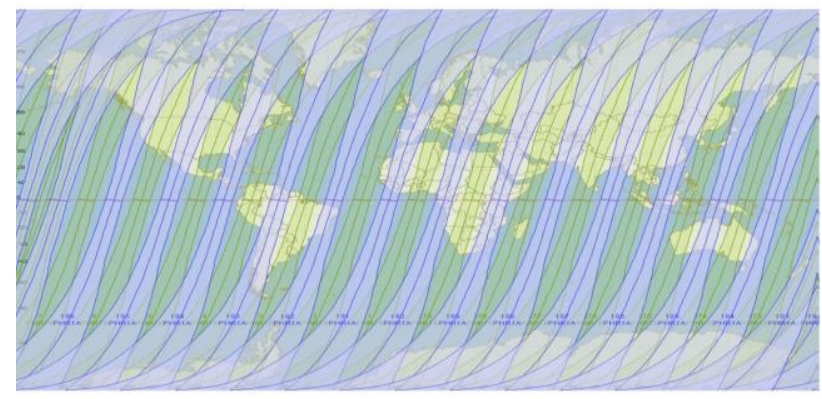

Figure 2. Visibility of Pléiades-1A \& 1B for one day $+/-30^{\circ}$ (ASTRIUM, 2012)

Pléiades satellite record multispectral and panchromatic information. Multispectral images contain the red, green, blue and near infrared band with a resolution of $2.0 \mathrm{~m}$ whereas the panchromatic band has a spatial resolution of $0.5 \mathrm{~m}$. Rated as a very high resolution (VHR) satellite, a large variety of applications is feasible such as hazard mapping, detailed landuse classifications or urban mapping.

Pléiades satellites are able to collect images in tri-stereo acquisition mode, meaning that three images are recorded from the same area within one pass (see Figure 3).

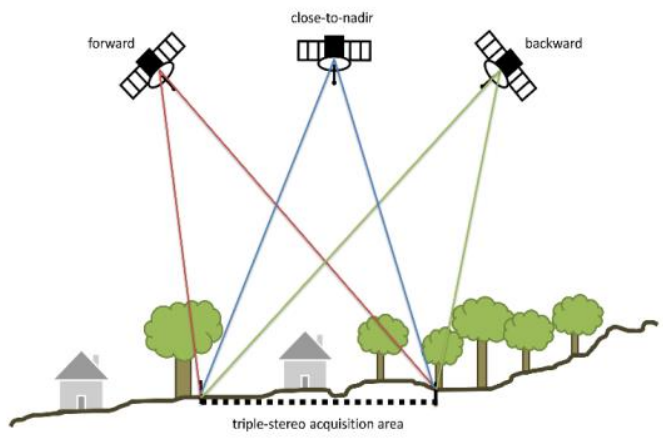

Figure 3. Tri-Stereo acquisition mode

As compared to stereo acquisition, which is the minimum configuration to extract 3D information, tri-stereo data records an image from close-to-nadir acquisition angle. This is of great advantage for urban areas or mountainous regions, because information from areas with urban canyons can be extracted well whereas they cannot be seen with mere stereo images. Moreover, it is of particular importance for urban areas, that the Base over Height $(\mathrm{B} / \mathrm{H})$ ratio is chosen according to the characteristics of the study area before data acquisition. The $\mathrm{B} / \mathrm{H}$ ratio indicates the stereoscopic capacity of a stereo image pair. If a high $\mathrm{B} / \mathrm{H}$ ratio is chosen, areas of urban valleys could not be seen (see Figure 4).
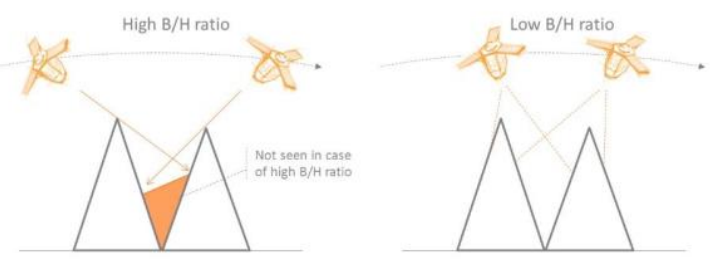

Figure 4. Comparison between a high and low $\mathrm{B} / \mathrm{H}$ ratio (ASTRIUM, 2012)

ASTRIUM (2012) recommends a value between 0.4 and 0.7 for DSM generation and a value smaller than 0.4 for urban area and mountainous regions.

For the study, there has been used tri-stereo satellite imagery acquired on 1 September 2015 for the whole extent of the study area including red, green, blue, NIR and panchromatic band.

\subsection{Stereo Matching}

For the generation of the digital surface model (DSM) there is used a stereo matching algorithm called Semi-Global Matching (SGM). To run the SGM algorithm, it is necessary to have a known epipolar geometry (see Figure 5). 


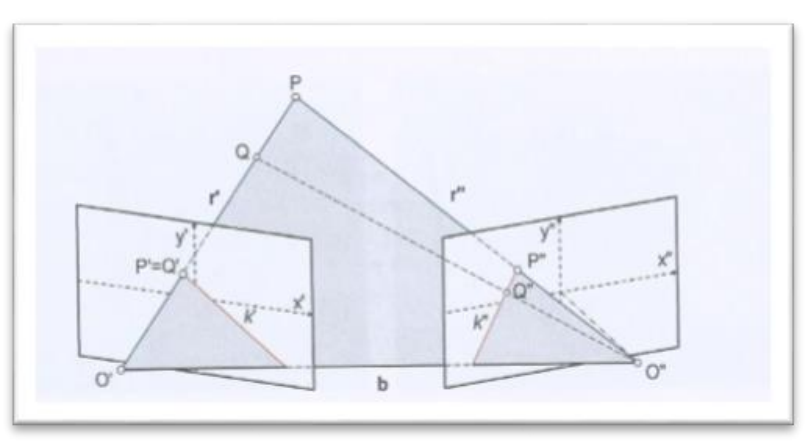

Figure 5. Epipolar Geometry (Luhmann et al., 2014)

The perspective centre $O$ of the camera lies on the same line with the image point $Q$ and the respective point of the recorded object $P$. Having two images, there are also two lines $r$ ' and $r$ '. These two lines form together with the line between the two camera projection centres the epipolar plane which intersects the image planes in a line, called epipolar line. The image point and also the corresponding point lie on this epipolar line. Having a known epipolar geometry reduces the search space because the corresponding point has to be searched only along the epipolar line and not in the whole image. The SGM algorithm as an energy function which should be minimal:

$$
\mathrm{E}(\mathrm{D})=\sum_{P} C\left(p, d_{p}\right)+\sum_{q \epsilon n_{p}} P\left\lfloor\left|d_{p}-d_{q}\right| \geq 1\right]
$$

where $\quad \mathrm{p}=$ pixel $\mathrm{p}$

$\mathrm{d}=$ distance

$\mathrm{N}_{\mathrm{p}}=$ neighbourhood of pixel $\mathrm{p}$

$\mathrm{q}=$ all pixels from the neighbourhood $\mathrm{N}_{\mathrm{p} \text { of }}$ pixel $\mathrm{p}$

Luhmann et al., 2014

This function consists of two parts: the cost function $C$ and the penalty term $P$. The cost function defines the cost needed for matching a pixel with corresponding pixel at a distance $d$ along the epipolar line. The penalty term penalises disparities of all pixels from the neighbourhood of the pixel $p$ which is larger than 1. This results in an exponential growth of computational effort with an increasing size of input data. For this reason, Hirschmueller (2005) did not use the global matching approach but introduced the SGM which decreases the computational effort by using a combination of local and global matching. For SGM eight linear paths through the image get calculated running radially and symmetrically from image boundaries meeting at pixel $p$. Finally, the disparity with the least cost is chosen (Hirschmueller, 2011).

\subsection{DSM generation and object extraction}

This SGM algorithm is meanwhile also implemented to several GIS software such as ERDAS Imagine and usable for satellite imagery. This software has been also used for the DSM generation of this study. After image block creation and triangulation the SGM algorithm has run resulting in three point clouds, one for each image pair. The SGM calculation in ERDAS Imagine can be only calculated for image pairs but not for three images in one step. After the conversion of the point cloud to a raster grid, the DSM derived from Pléiades tri-stereo imagery can be used.

Having height information and spectral information from the same sensor is advantageous because it has been recorded at the same time and the same spectral resolution. This data is then used to extract different objects of the urban areas, such as buildings or vegetation, based on the height value, spectral information and/or shape and neighbourhood relations. The objects have been extracted with an object-based approach in eCognition software. There has been created a ruleset to classify objects according to their shape, spectral values and relation to other objects.

\section{RESULTS}

\subsection{DSM Evaluation}

The quality of the DSM derived from Pléiades tri-stereo satellite imagery is quite good overall. Some outliers occur in the data set due to shadowed areas and high disparity differences. Data gaps, where the algorithm did not find corresponding points, lead to inaccuracies due to interpolation. Comparing the DSM derived from Pléiades with a DSM based on LiDAR data, an overall accuracy of around $1 \mathrm{~m}$ has been achieved, which is a satisfying result, particularly for urban areas. Calculated heights were also compared to height information of the Pléiades DSM with measured GPS points existing all over the study area. This comparison confirmed the accuracy of around $1 \mathrm{~m}$.

\subsection{3-D Objects}

The 3-dimensional objects are created in two steps: first of all the object extraction in eCognition based on spectral information, shape, object relations and/or height information. As a second step the height information has been added to the created objects to get a 3-dimensional image object.

Based this information, statistics are calculated on different levels such as the minimum and maximum height, as well as the volume of objects. True height values can be calculated and not just an estimation of values as e.g. in Shao et al., 2011. In urban areas we could apply different scales for the calculation, for example single buildings or trees, neighbourhoods, complete forests or urban parks. Furthermore, we could use the units of an aerial zoning plan to check for the compliance with height constraints per zone (see Figure 6).

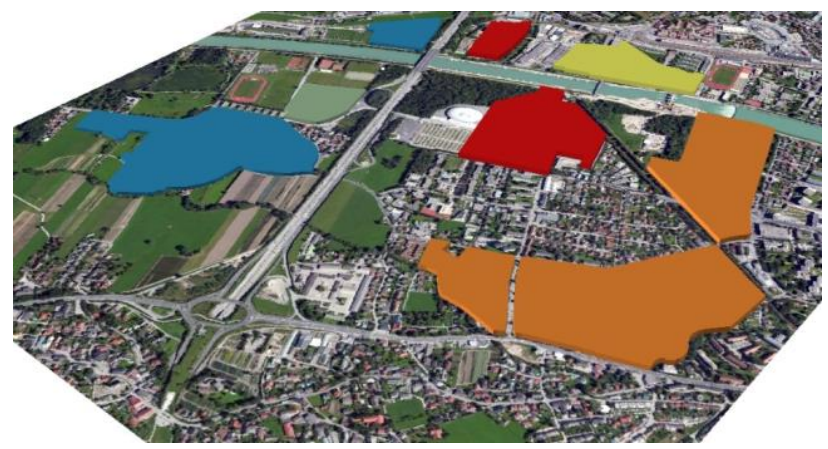

Figure 6. Statistical calculations for units of the aerial zoning plan 
Within the scope of this study, there has been a calculation of statistics which includes the minimum, mean and maximum height of an object and the volume of objects. The following levels of urban objects have been used: single object (single building, tree), building blocks, neighbourhood (neighbouring objects), urban parks and the aerial zoning plan.

Moreover, the height information is not only used to calculate statistics in urban areas but is also helpful in the classification of a scene. A classification is refined by an additional feature: the height of an object. It also works good to use the elevation data for the classification and classify objects according to their heights. Objects of similar heights are then grouped together and form specific areas such as the inner city with a larger building height value in comparison with a lower height value for bushes.

Urban planners can use this information for planning purposes and to monitor dynamics in urban areas. Furthermore, these 3dimensional objects can be used in urban context to monitor changes in the urban areas. Updating the Pléiades data - the spectral information as well as the elevation data - could help to detect changes in the city. To keep comparability it would be advantageous to always use data from the same sensor, such as Pléiades.

Using Pléiades tri-stereo satellite imagery does not have only advantages in this context but could also cause some inaccuracies. In comparison to laser scanning data, Pleíades has a resolution of $0.5 \mathrm{~m}$ in the panchromatic band, whereas LiDAR data reaches much higher resolution nowadays. Furthermore, by taking the calculation of vegetation volumes as an example, the DSM from Pléiades only gives the information on the tree crown which is visible from the perspective of the sensor. Tree trunks could not be seen in this kind of data.

\section{CONCLUSIONS}

Using Pléiades tri-stereo satellite imagery for 3-D object extraction could have different advantages: height and thematic imformation can be extracted simultaneous. This data is good for synergistic use because it comes from the same sensor and there is no time gap in between. If the spatial resolution is sufficient for the respective purpose it could be a good alternative to laser scanning data because it saves costs and the acquisition effort is not that high. Additionally, satellite data can be updated regularly under low costs which make them very useful in urban context, e.g. for urban planning purposes.

For the future, it is important to improve the result of the Pléiades DSM calculated by SGM and develop filtering methods which filter all the outliers and errors from the point cloud. Furthermore, it could be a possibility to use mono satellite imagery from the archive and match multiple mono scenes to get the same result. With this method it is possible to create DSMs from multiple previous mono acquisitions which could be, in comparison with newly ordered tri-stereo data, a good data base for the monitoring of urban dynamics.

\section{REFERENCES}

- ASTRIUM (2012), Pléiades Imagery, User Guide. Technical Reference.

- GEHRKE, S., MORIN, K., DOWNEY, M., BOEHRER, N., \& FUCHS, T. (2010), Semi-global matching: An alternative to LiDAR for DSM generation. In Proceedings of the 2010 Canadian Geomatics Conference and Symposium of Commission I.

- HIRSCHMUELLER, H. (2005), Accurate and efficient stereo processing by semi-global matching and mutual information. In: 2005 IEEE Computer Society Conference on Computer Vision and Pattern Recognition (VCPR`05) (Vol. 2) 807-814.

- HIRSCHMUELLER, H. (2011), Semi-global matching-motivation, developments and applications. Photogrammetric Week, Wichmann/VDE Verlag, Berlin \& Offenbach, 173-182.

- LUHMANN, T.; ROBSON, S.; KYLE, S.; BOEHM, J. (2014), Close-Range Photogrammetry and 3D Imaging. 2nd Edition. Walter de Gruyter GmbH.

- SHAO, Y.; TAFF, G. N.; \& WALSH, S. J. (2011), Shadow detection and building-height estimation using IKONOS data. International journal of remote sensing, 32(22), 6929-6944.

- ZHOU, W. (2013), An object-based approach for urban land cover classification: integrating LiDAR height and intensity data. IEEE Geoscience and Remote Sensning Letters, 10(4), 928-931. 